\title{
$\rightarrow$ \\ Laparoscopic cholecystectomy and outpatient surgery
}

In recent years it may be safely stated that a major change in the understanding of surgery has taken place with laparoscopic surgery in the late 1980s. Indeed, the linking of technologic development circumstances to the will of a number of surgeons to offer less damaging surgery led to the notion of "minimally aggressive surgery", in which laparoscopic surgery is paradigmatic.

Laparoscopic surgery entailed a number of nowadays universally accepted benefits for patients. In fact, initial distrust regarding this way of surgery has led to a careful analysis of its results which we are convinced was unparalleled before for surgical techniques. Thus, not only has laparoscopy advanced in itself, but traditional techniques have also benefited from greater rigor in outcome studies, and healthy competition has shown up regarding "lesser invasion" with increasingly small incisions, and shorter stays and postoperative periods, all of which represents great value for patients.

Regarding laparoscopic cholecystectomy (LC), this procedure evolved in 6 years -from the time is was first used back in 1987- to become the gold-standard in the treatment of symptomatic cholethiasis. However, it should be noted that scientific evidence supporting its superiority versus traditional cholecystectomy as based upon controlled randomized studies is pretty scant, regardless its wide acceptance by the health-care community and patients as well.

Although many comparative studies exist on all sorts of partial aspects that clearly reveal the benefits of LC -from classic morbidity and mortality, and hospital stay studies to sophisticated studies on- say -post-procedural immune response- only two prospective, randomized, single-blind studies compared laparoscopic versus open cholescystectomy $(1,2)$, and none showed clearly significant differences for either procedure. The importance of these studies is relative when compared to downpouring experience pointing that laparoscopy provides a far more benign immediate postoperative time. We should not be oblivious regarding the fact that the so-called "surgeon factor" may never be blind -and is difficult to assess- and therefore double-blind studies are not feasible.

This evidence regarding a generally benign postoperative period led to consider that this technique could be performed on an outpatient basis with no overnight hospital stay, in a search for cost savings, either institutional or private, in each procedure. We would like to stop here at any rate to superficially analyze concept differences amongst cultures and health-care models with respect to major outpatient surgery (MOS).

The North American model mainly attempts to reduce costs per procedure, which in financial terms will indirectly impact on users as regards medical insurance costs and of course by making private procedures cheaper. To this end hospitals 
usually rely on nearby hotels where patients may stay following hospital discharge, should they wish so, so that they may be cared for by their surgeon if needed.

The same goes for private surgery, but regarding public health-care, a universal, free-of-charge model such as the Spanish one is mainly concerned in hospital stay reduction in addition to reduced costs per procedure, in an attempt to free hospital beds for other procedures and send patients home upon surgery completion. Besides, health-care sectorialization renders distances to hospitals significant in the rural setting, and the sector cared for by a given hospital may include urban areas with deficient housing or serious social-financial difficulties. All these reasons together with a lack of perception of financial benefits by patients render day-care surgery more difficult to implement and their presence uneven.

Regarding other surgical procedures habitually included in MOS, LC has the differential characteristic of being a technique requiring general anesthesia for an approach of the peritoneal cavity. Thus, patients who are at high-risk regarding anesthesia may not be included, where in addition to potential surgical complications an eye must be kept on potential anesthesia-related complications. On the other hand, potential cholecystitis or post-cholecystitis sequelae -which are often hard to predict- may render this procedure more complex.

In the present issue of our REED, two papers are published that relate to different outpatient LC-related topics. Whereas Vieira et al. (3) refer to the setting up of an outpatient LC program, Planells et al. (4) focus on patients with non-lithiasic cholecystopathies who undergo daily-care LC. The latter authors reported in 2001 on their experience along 4 years of outpatient LC in this same journal (5), and were understandably considered a pioneer group for LC in MOS in our country.

On the topic of alithiasic bladder conditions we will merely point out that, as in the authors' experience, bladder conditions that are considered alithiasic very commonly share the etiology of lithiasic disease, be it because of the presence of undetected microlithiasis or cholesterolosis. An experience that is not uncommon in daily practice involves patients who are admitted with acute pancreatitis in whom no lithiasic condition is seen, and who are therefore referred to the Gastroenterology Unit for an etiologic study. After a variable lapse of time, and following new pancreatitis episodes, a percentage of these patients are sent back upon detection or suspicion of lithiasis in tests performed. Therefore, as Shaffer puts it (6), these patients must be separated from those suffering from true acalculous biliary dyskinesia, a distinct condition which commonly includes Oddi's sphincter dysfunction, which in turn may lead to failed cholecystectomy management. The problem is that bladder and bile duct motility is difficult to acknowledge in daily practice (7), and disorders may be in combination with other gastrointestinal motility abnormalities (8).

Anyway, patients preoperatively labeled as having alithiasic cholecystopathies are overall excellent candidates to outpatient LC, at least regarding a lower likeliness of complicated surgical procedures.

Overall experience regarding LC in MOS is consistent with observations in other techniques, that is, that greater expertise in a procedure and its common daily-care use improve results. Recently, Oteiza et al. reported on an interesting study that compared hernia surgery outcomes between surgeons in one department (9). One group performed the procedure habitually and on an outpatient basis in most cases, whereas the other group performed the technique rather sporadically among their scheduled standard procedures. Outcomes were significantly better in the first group, whose procedures were seen to be also more deeply standardized.

This is, in our view, a scarcely mentioned benefit of daily-care LC, since a signi- 
ficant improvement in procedural quality most likely occurs through the specialization of team members and the careful care needed for early discharge.

But quality management is as important regarding "offered quality" as regarding "perceived quality", and this is often harder to convey so that early discharged patients perceive it, be it because of fear even in the absence of complications or because of a magnification of events that obviously might also have developed should the patient have stayed in hospital. In this regard information provided by Planells et al. in a paper published in 2002 (10) is in our view highly significant, as it states that $20 \%$ of their patients were very much in worry during their first night at home even when they had accepted the procedure and were satisfied with it.

In any case LC is no doubt an excellent procedure, currently the gold-standard in the treatment of symptomatic cholelithiasis, which may be performed as MOS in a high number of patients. For a group of patients it will not be the suitable technique due to medical reasons; for another, much more varying group of patients it will not be feasible because of social reasons. Similarly, a small number of failures from intra- or postoperative complications exist, which will diminish as experience is gained in both patient selection and surgical, anesthetic and nursing management scheduling. Its feasibility has been demonstrated in all settings and differing countries with rather similar results (10-14).

The accounting peculiarities of the Spanish health-care system makes it very difficult to quantify presumed savings versus inpatient surgery, since the cost needed to set up a home care system infrastructure and that of readmissions should have to be subtracted from reduced costs per bed and day. It is however important to consider this procedure's frequency, which has notably increased during the last years as a result of improved diagnostic procedures and a greater predisposition of patients to be operated on. It has been estimated that the number of procedures have increased from 500,000/year to above 800,000/year in the United States during the last decade; considering the population of the European Union, only in the Western, developed hemisphere the number of procedures is much higher than one million procedures per year. Thus, savings incurred will have a tremendous impact on health-care expense.

However, in the Spanish health-care system, which is in need of public beds in most regions, reductions in the hospitalization stay are also important besides savings, so that beds may be put to good use for other patients, which may be achieved via MOS programs including well trained teams in outpatient LC for feasible cases.

\author{
P. Rico Selas and A. Calle Santiuste \\ Service of General, Digestive and Transplant Surgery. \\ Hospital 12 de Octubre. Madrid. Spain
}

\title{
REFERENCES
}

1. Majeed AW, Troy G, Nicholl JP, Smythe A, Reed MW, Stoddard CJ, Peacock J, Johnson AG. Randomized, prospective, single-blind comparison of laparoscopic versus small-incision cholecistectomy. Lancet 1996; 347: 989-94

2. Ros A, Gustafsson L, Krook H, Norgren CE, Thorell A, Wallin G, et al. Laparoscopic cholecistectomy versus mini-laparotomy cholecistectomy. A prospective, randomized, single-blind study. Ann Surg 2001; 234: 741-9. 
3. Martínez Vieira A, Docobo Durántez F, Mena Robles J, Durán Ferreras I, Vázquez Monchul J, López Bernal, et al. Laparoscopic cholecystectomy in the treatment of biliary lithiasis: outpatient surgery or short stay unit? Rev Esp Enferm Dig 2004; 96 (7): 452-9.

4. Planells Roig M, Bueno Lledó J, Sanahuja Santafé A, García Espinosa R. Quality of life (GIQLI) and laparoscopic cholecystectomy usefulness in patients with gallbladder dysfunction or chronic non-lithiasic biliary pain (chronic acalculous cholecystitis). Rev Esp Enferm Dig 2004; 96 (7): 442-51.

5. Serralta A, García-Espinosa R, Martínez-Casañ P, Hoyas L, Planells M. Cuatro años de experiencia en colecistectomía laparoscópica ambulatoria. Rev Esp Enferm Dig 2001; 93: 207-10.

6. Shaffer E. Acalculous biliary pain: new concepts for an old entity. Dig Liver Dis 2003; 35 (Supl. 3): S20-5.

7. Lancini A, Lanzarotto F, Baisini O, Amato M, Benini F. Value of measuring gallbladder motility in clinical practice. Dig Liver Dis 2003; 35 (Supl. 3): S46-50.

8. Colecchia A, Sandri L, Staniscia T, Vestito A, Capodicasa S, Portincasa P, et al. Gallbladder motility and functional disorders. Dig Liver Dis 2003; 35 (Supl. 3): S30-4.

9. Oteiza F, Ortiz H, Ciga MA. Tratamiento de la hernia inguinocrural. Resultados comparativos entre una unidad de pared abdominal y un servicio de cirugía general. Cir Esp 2004; 75: 146-8.

10. Planells M, Sánchez A, Sanhuja A, Bueno J, Serralta A, García-Espinosa R, et al. Gestión de la calidad total en colecistectomía laparoscópica. Calidad asistencial y calidad percibida en colecistectomía laparoscópica ambulatoria. Rev Esp Enferm Dig 2002; 94: 319-25.

11. Leeder PC, Matthews T, Krzeminska K, Dehn TC. Routine day-case laparoscopic cholecistectomy. Br J Surg 2004; 91: 312-6.

12. Bal S, Reddy LG, Parshad R, Guleria R, Kashyap L. Feasibility and safety of day care laparoscopic cholecistectomy in a developing country. Postgrad Med J 2003; 79: 284-8.

13. Barthelsson C, Lutzen K, Anderberg B, Nordstrom G. Patients' experiences of laparoscopic cholecistectomy in day surgery. J Clin Nurs 2003; 12: 253-9.

14. Lau H, Brooks DC. Contemporary outcomes of ambulatory laparoscopic cholecistectomy in a major teaching hospital. World J Surg 2002; 26: 1117-21.

\section{Colecistectomía laparoscópica y cirugía ambulatoria}

Podemos afirmar que el mayor cambio en la forma de entender la cirugía, en los últimos años, se ha dado con la aparición de la cirugía laparoscópica a finales de la década de los 80. En efecto, la concatenación de una serie de circunstancias de desarrollo tecnológico, junto con las inquietudes de muchos cirujanos por ofrecer una cirugía menos lesiva, sirvieron para crear el concepto de "cirugía mínimamente agresiva" de la cual el paradigma es la cirugía laparoscópica.

La cirugía laparoscópica ha facilitado una serie de ventajas para los pacientes que hoy son aceptadas universalmente. Precisamente el recelo inicial hacia esta forma de abordar la cirugía ha hecho que sus resultados hayan sido analizados minuciosamente de una forma que, nos atreveríamos a asegurar, no había sucedido anteriormente con ninguna técnica quirúrgica. De esta forma no solamente se ha producido un avance con la laparoscopia en sí misma sino que las técnicas tradicionales también se han beneficiado de un mayor rigor en los estudios de los resultados, se ha producido una sana competencia en cuanto a la "menor invasión" con incisiones más pequeñas, estancias y tiempos postoperatorios más ajustados, etc., concurriendo todo ello en un beneficio para los pacientes.

En el caso concreto de la colecistectomía laparoscópica (CL), es llamativo que en el plazo de 6 años, a partir de realizarse por vez primera en 1987, pasara a ser considerada el "gold-standard" en el tratamiento de la colelitiasis sintomática. Sin embargo, hemos de reconocer que, junto a esta aceptación por parte no sólo del 
mundo sanitario sino de los pacientes que la demandan, la evidencia científica, en cuanto a la demostración de su superioridad frente a la colecistectomía tradicional, basada en estudios controlados randomizados es bastante escasa.

En efecto, mientras existen infinidad de estudios comparativos sobre aspectos parciales de todo tipo, que ponen de manifiesto las ventajas de la CL, pasando por los estudios clásicos de morbimortalidad y la estancia hospitalaria e incluyendo estudios sofisticados, por ejemplo sobre la respuesta inmunitaria del organismo postintervención, solamente existen dos estudios prospectivos, randomizados, simple ciego que comparen de forma global la colecistectomía laparoscópica con la técnica abierta $(1,2)$ y en ninguno de los dos se observan diferencias claramente significativas a favor de una u otra técnica. La importancia de estos trabajos es relativa frente al aluvión de experiencia a favor de que la laparoscopia proporciona un postoperatorio inmediato más benigno. Tampoco podemos olvidar que, en estudios sobre cirugía, el "factor cirujano", además de difícil de evaluar, nunca puede ser "ciego" por lo que no son factibles estudios "doble-ciego".

Es esta evidencia de un postoperatorio, en general, tan benigno lo que ha llevado a plantear la posibilidad de su realización de forma ambulatoria, esto es sin ingreso hospitalario nocturno, en busca de un ahorro en costes, institucionales o particulares, por procedimiento. Aquí, en cualquier caso, nos gustaría detenernos un momento para analizar muy someramente las diferencias conceptuales entre las diferentes culturas y los diferentes modelos sanitarios frente a la cirugía mayor ambulatoria (CMA).

En el modelo sanitario norteamericano se trata fundamentalmente de reducir costes por proceso que, en términos económicos, repercutirán indirectamente en los usuarios en forma del coste de los seguros médicos y, por supuesto, en forma de un abaratamiento de las intervenciones privadas. Para ello los hospitales a menudo cuentan con hoteles-residencias cercanas donde los pacientes pueden permanecer, tras el alta hospitalaria, si lo desean, para ser asistidos por su cirujano en caso de necesidad.

En los casos de cirugía privada el caso sería el mismo anteriormente expuesto pero, en la Sanidad Pública, en un modelo de asistencia universal y gratuita como el español, además de la reducción de costes por proceso, se trata fundamentalmente de la reducción de estancias hospitalarias para liberar camas para otros usos y los pacientes vuelven a su domicilio tras la intervención. Por otro lado la sectorización de la asistencia hace que, en el medio rural, las distancias puedan ser importantes respecto al centro hospitalario y, en el medio urbano, puede que el sector de atención del hospital cubra zonas con deficiencias en las viviendas o con importantes problemas socioeconómicos. Todas estas razones, unidas a la no-percepción por parte de los pacientes de ventajas en términos económicos de la cirugía ambulatoria, hacen más difícil su implantación y que esta sea muy desigual.

Respecto a otros procedimientos quirúrgicos que se incluyen habitualmente en CMA, la CL presenta como característica diferencial el ser una técnica que precisa anestesia general en la cual se aborda la cavidad peritoneal. Esto implica que no puedan ser incluidos los pacientes con un riesgo anestésico alto y que, además de la prevención de las complicaciones puramente quirúrgicas haya que estar atentos a la eventualidad de complicaciones anestésicas. Por otro lado la posibilidad de colecistitis o de secuelas postcolecistitis, no siempre previsibles, puede hacer que el procedimiento sea más complicado de lo habitual.

En el presente número de nuestra revista se publican dos trabajos sobre temas distintos relacionados con la CL ambulatoria. Mientras que Vieira et al. (3) se refie- 
ren a la implantación de un programa de CL ambulatoria, Planells et al. (4) se centran en pacientes con colecistopatías no litiásicas tratados con CL ambulatoria. Estos últimos autores publicaron en 2001 su experiencia en 4 años de CL ambulatoria en esta misma revista (5) siendo considerado con justicia uno de los grupos pioneros en nuestro país en la realización de CL en CMA.

Respecto al tema de la patología vesicular alitiásica, sin entrar en otro tipo de consideraciones, únicamente señalar que, como en la experiencia de los autores, la patología vesicular considerada "alitiásica" muy frecuentemente tiene la misma etiología que la litiásica, sea por la presencia de microlitiasis no detectada o de colesterolosis. Una experiencia que no es infrecuente en la práctica clínica diaria es la de pacientes que ingresan por pancreatitis aguda en los cuales no se identifica patología litiásica y son derivados al servicio de Gastroenterología para su estudio de otras posibles etiologías. Tras un tiempo variable, y algún episodio más de pancreatitis, un porcentaje de estos pacientes nos es devuelto tras detectarse o sospecharse una litiasis en las exploraciones practicadas. Por tanto, como opina Shaffer (6), estos pacientes deben ser separados de aquellos en los que se da una auténtica disquinesia biliar acalculosa que es una entidad distinta en la que a menudo está implicada una disfunción del esfínter de Oddi que puede ser causa del fracaso en el tratamiento con colecistectomía. El problema es que la motilidad de la vesícula y las vías biliares es muy difícil en la práctica clínica (7) y que los trastornos pueden estar combinados con otras anomalías de la motilidad digestiva (8).

En cualquier caso, los pacientes etiquetados preoperatoriamente como colecistopatías alitiásicas son, en general, unos excelentes candidatos a CL ambulatoria al menos en lo que se refiere a una menor probabilidad de un procedimiento quirúrgico complicado.

La experiencia general respecto a la CL en CMA es concordante con lo observado en cualquier otra técnica, esto es que la mayor expertización en un procedimiento y en su realización habitual ambulatoria hace que mejoren los resultados. Recientemente Oteiza et al. publicaban un estudio interesante en el que comparaban los resultados en cirugía de hernia entre cirujanos del mismo servicio (9). Un primer grupo realizaba la técnica de manera habitual y en cirugía ambulatoria en la mayor parte de los casos y el otro grupo la realizaban más esporádicamente dentro de sus intervenciones programadas normales. Los resultados eran muy significativamente mejores en los del primer grupo además de demostrarse que los procedimientos estaban más estandarizados.

Esta es una ventaja que creo poco mencionada de la CL ambulatoria ya que, a través de la especialización de los miembros del equipo que interviene y de los cuidados minuciosos necesarios para que pueda ser llevada a cabo un alta precoz, muy probablemente se produce una mejora sustancial en la calidad del proceso realizado.

Pero en una gestión total de la calidad tan importante como la "calidad ofertada" es la "calidad percibida" y, en muchos casos es más difícil transmitirla para que la perciban los pacientes con alta precoz bien sea por el temor generado aún en ausencia de complicaciones o por la magnificación que se puede producir ante cualquier eventualidad que, obviamente, se hubiera producido de la misma forma permaneciendo en el hospital. En este sentido nos parece muy demostrativo el dato que dan Planells et al. en otro artículo publicado en 2002 (10) en el que refiere que un $20 \%$ de sus pacientes, aun aceptando el procedimiento y estando satisfechos con el mismo, referían haber estado intranquilos en la primera noche en su domicilio.

En cualquier caso, creemos que no cabe ninguna duda de que la CL es un procedimiento excelente, actual "gold-standard" en el tratamiento de la colelitiasis sinto- 
mática y que, en un alto número de pacientes, puede ser realizado en CMA. Es preciso contar con un número de casos en que no parece la táctica más idónea por razones médicas y con otro, que será mucho más variable según los ámbitos de actuación, en que no será posible por razones sociales. Asimismo hay que contar con un pequeño porcentaje de fracasos por complicación intra o postoperatoria que se irá reduciendo conforme se adquiera experiencia tanto en la selección de los pacientes como en la planificación del tratamiento quirúrgico, anestésico y de enfermería. Se ha demostrado su factibilidad en todos los ámbitos y distintos países con resultados bastante parejos (10-14).

Las peculiaridades contables del sistema de salud español hace que sea muy difícil cuantificar el supuesto ahorro que se produce respecto a la cirugía con ingreso ya que de la reducción del coste por cama y día habría que restar el coste que precisa la creación de una infraestructura de atención a los pacientes en su domicilio y el generado por las readmisiones. Pero es importante considerar la frecuencia de la intervención, notablemente incrementada en los últimos años, debido a la mejora en los procedimientos diagnósticos y a la mayor predisposición de los pacientes a intervenirse. Se calcula que, en EE.UU., se ha pasado de 500.000 intervenciones/año a más de 800.000 en la última década por lo que, teniendo en cuenta la población de la Unión Europea, solamente en el mundo desarrollado occidental se sobrepasa con mucho el millón de intervenciones al año. Por tanto cualquier ahorro tendrá una repercusión tremendamente importante en el gasto sanitario.

Pero en un sistema sanitario como el español, con necesidades de camas públicas en la mayoría de las regiones, además del ahorro es también importante la reducción de estancias, para dedicar las camas a otros usos, que puede conseguirse con programas de CMA que incluyan equipos entrenados en la realización de CL ambulatoria en los casos en que sea factible.

\author{
P. Rico Selas y A. Calle Santiuste \\ Servicio de Cirugía General, Digestiva y Trasplantes. \\ Hospital 12 de Octubre. Madrid
}

